\title{
Nanofiber-Mediated Interaction of Light with Cold Atoms
}

\author{
A.S. Sheremet ${ }^{1,2}$ a, B. Gauraud ${ }^{3}$, N. Corzo-Trejo ${ }^{3}$, J. Laurat ${ }^{3}$, and D.V. Kupriyanov ${ }^{2}$ \\ ${ }^{1}$ Russian Quantum Center, 143025 Skolkovo, Moscow Region, Novaya str.100a, Russia \\ ${ }^{2}$ Saint Petersburg Polytechnic University, 195251 Saint-Petersburg, Polytechnicheskaya str., 29, Russia \\ ${ }^{3}$ Laboratoire Kastler Brossel, Universite Pierre et Marie Curie, 75005 Paris, 4 place Jussieu, France
}

\begin{abstract}
Light-matter interaction processes are extremely attractive for quantum information problems, where one of the main goals consists in a quantum state of light mapping into a collective atomic state. By analogy with the Purcell effect, which exhibits in the presence of a resonator, enhancement of light-matter interaction can be achieved by using a nanofiber [1-2]. In this work we present a theoretical description of light interaction with a cold atomic ensemble mediated the nanofiber, which lies in a base of the experimental realization of a quantum memory protocol [3].
\end{abstract}

Light-matter interaction plays an important role in the implementation of unique application of quantum optics, such as quantum memory protocol. In recent years, the physical implementation of quantum interfaces between light and matter in free space has initiated a very active research. In spite of significant advances, the efficiency of demonstrated quantum memory protocols is not extremely high, which is issued with problems of achievement of high-efficient light-matter coupling.

Interfacing guided light with atoms has therefore been foreseen as a promising alternative approach, enabling to increase the light-matter coupling as well as to obtain a large optical depth. Pioneering works on the theoretical investigation of interaction processes of atoms with guided light were performed by group of K. Hakuta [1-2]. A significant role in such kind of interaction processes belongs to the evanescent field, which appears due to a small diameter of the nanofiber (less then light wavelength) that leads to more effective light-matter coupling in the evanescent field area.

In the present work we consider experimental demonstration and theoretical description of the quantum memory protocol for the nanofiber guided light interfacing with $133 \mathrm{Cs}$ atoms, cooled in a magneto-optical trap [3]. The nanofiber guided signal field interacts with atoms on atomic transition of D2-line. The control field is applied on resonance to atomic transition and creates the transparency window in accordance with the electromagnetically induced transparency (EIT) mechanism. We consider the light interaction process with all hyperfine atomic structure and discuss the influence of the nanofiber on the light interaction process with the atomic system.

\section{References}

1. F. Le Kien, S. Dutta Gupta, V.I. Balykin, and K. Hakuta, Phys. Rev. A 72, 032509 (2005)

2. F. Le Kien, V.I. Balykin, and K. Hakuta, Phys. Rev. A 73, 013819 (2006)

3. B. Gouraud, D. Maxein, A. Nicolas, O. Morin, J. Laurat, Phys. Rev. Lett. 114, 180503 (2015)

${ }^{\mathrm{a}}$ Corresponding author: sheremet.alexandra@gmail.com 